\title{
PRODUÇÃO DE LIPÍDEOS A PARTIR DE DIFERENTES ESPÉCIES DE MICROALGAS
}

\author{
R. R. DIAS ${ }^{1}$, S. F. SIQUEIRA ${ }^{1}$, M. M. MARONEZE ${ }^{1}$, L. Q. ZEPKA ${ }^{1}$ e E. JACOB-LOPES ${ }^{1}$ \\ ${ }^{1}$ Universidade Federal de Santa Maria, Depto. de Tecnologia e Ciência de Alimentos, Av. \\ Roraima, 1000, CEP 97105-900, Santa Maria, Rio Grande do Sul \\ E-mail para contato: jacoblopes@pq.cnpq.br
}

\begin{abstract}
RESUMO - O objetivo do trabalho foi avaliar a produção de lipídeos pelas microalgas Chlorella vulgaris, Scenedesmus obliquus, Aphanotece microscopica Nägeli e Phormidium autumnale. Os cultivos foram desenvolvidos em um biorreator do tipo coluna de bolhas. O sistema de dispersão de gases do reator constituiu em um difusor de ar de $1,5 \mathrm{~cm}$ localizado no centro da base da coluna. A concentração inicial de células foi de $100 \mathrm{mg} / \mathrm{L}$, operando a uma temperatura de $26^{\circ} \mathrm{C}$, com luminosidade de $150 \mu \mathrm{mol} . \mathrm{m}^{-2} \mathrm{~s}^{-1}$ e aeração continua de $1 \mathrm{VVM}$ com a injeção de ar enriquecido com $15 \%$ de dióxido de carbono. A partir da fração lipídica da biomassa seca, o perfil lipídico foi avaliado. Dentre as espécies testadas a Phormidium autumnale apresentou maior produtividade lipídica (3,8 $\mathrm{mg} / \mathrm{L} . \mathrm{h})$ e teor lipídico $(27,0 \%)$, demostrando ser um potencial para a produção de lipídeos.
\end{abstract}

\section{INTRODUÇÃO}

As microalgas são uns dos microrganismos mais antigos existentes e apresentam-se em formas unicelulares ou em cadeias. A composição das microalgas em ácidos graxos de cadeias longas, principalmente insaturadas como Omega-3 e Omega-6, além da alta concentração de proteínas e carboidratos torna-as fontes ideais para o preparo de alimentos funcionais, aditivos de alimentos, entre outros (Mariano et al., 2009). Elas sintetizam e acumulam quantidades substanciais de lipídeos, que não só varia entre espécies, mas podem ser moduladas por fatores bióticos e abióticos.

Os produtos lipídicos de microalgas incluem lipídeos, ácidos graxos, pigmentos solúveis em gordura e vitaminas lipossolúveis com potenciais aplicações em energia, alimentos, cosméticos e produtos farmacêuticos (Harwatia et al., 2012). Os lipídeos microalgais ganharam atenção nas últimas décadas devido ao seu potencial de aplicação em muitas áreas. Contudo, o sucesso de qualquer processo fundamentado em algas é dependente de dois fatores-chave; alta produtividade e qualidade de biomassa com baixo custo de produção (Maroneze et al., 2016).

A produtividade lipídica é o que gera maior impacto no rendimento, uma vez que a produtividade lipídica é o produto da produtividade em biomassa e conteúdo lipídico. Portanto, um processo ideal deve ser capaz de produzir lipídeos na maior produtividade com o maior teor de células lipídicas. Por ser uma fonte de recursos renováveis a produção de 
lipídeos, extraído de microalgas, tem representado uma grande promessa para aplicação industrial.

Neste sentido, o objetivo do trabalho foi avaliar a produção de lipídeos a partir de diferentes espécies de microalgas.

\section{MATERIAIS E MÉTODOS}

\subsection{Microrganismo e Meio de Cultura}

As microalgas avaliadas foram Chlorella vulgaris, Scenedesmus obliquus, Aphanotece microscopica Nägeli e Phormidium autumnale. As culturas foram mantidas em meio sintético BGN (Rippka et al., 1979). As condições de manutenção usadas foram $26^{\circ} \mathrm{C}$, intensidade luminosa constante de $150 \mu \mathrm{molm}^{-2} \mathrm{~s}^{-1}$ e aeração constante.

\subsection{Meio de Cultura}

Utilizou-se o meio sintético BGN (Rippka et al., 1979). A composição química do BGN é: $\mathrm{K}_{2} \mathrm{HPO}_{4}\left(0,03 \mathrm{~g} \cdot \mathrm{L}^{-1}\right), \mathrm{MgSO}_{4}\left(0,075 \mathrm{~g} \cdot \mathrm{L}^{-1}\right), \mathrm{CaC}_{12} \cdot 2 \mathrm{H}_{2} \mathrm{O}\left(0,036 \mathrm{~g} . \mathrm{L}^{-1}\right)$, citrato de amônio e ferro $\left(0,0006 \mathrm{~g} . \mathrm{L}^{-1}\right), \mathrm{Na}_{2}$ EDTA $\left(0,001 \mathrm{~g} . \mathrm{L}^{-1}\right), \mathrm{NaCl}\left(0,00072 \mathrm{~g} . \mathrm{L}^{-1}\right), \mathrm{NaNO}_{3}\left(0,015 \mathrm{~g} . \mathrm{L}^{-1}\right)$, ácido cítrico $\left(0,0006 \mathrm{~g} . \mathrm{L}^{-1}\right), \mathrm{Na}_{2} \mathrm{CO}_{3}\left(1,5 \mathrm{~g} . \mathrm{L}^{-1}\right)$, metais traços: $\left[\mathrm{H}_{3} \mathrm{BO}_{3}\left(0,0028 \mathrm{~g} . \mathrm{L}^{-1}\right), \mathrm{MnC}_{12} \cdot 4 \mathrm{H}_{2} \mathrm{O}\right.$ $\left(0,0018 \mathrm{~g} . \mathrm{L}^{-1}\right), \mathrm{ZnSO}_{4} \cdot 7 \mathrm{H}_{2} \mathrm{O}\left(0,00022 \mathrm{~g} \cdot \mathrm{L}^{-1}\right), \mathrm{Na}_{2} \mathrm{MoO}_{4} \cdot 2 \mathrm{H}_{2} \mathrm{O}\left(0,00039 \mathrm{~g} . \mathrm{L}^{-1}\right), \mathrm{CoSO}_{4} \cdot 6 \mathrm{H}_{2} \mathrm{O}$ $\left(0,00004 \mathrm{~g} \cdot \mathrm{L}^{-1}\right)$.

\subsection{Biorreator}

Os experimentos foram realizados em biorreator de coluna de bolhas construído de vidro borossilicato com $0,5 \mathrm{~mm}$ de espessura, diâmetro interno de $6,5 \mathrm{~cm}$, altura de $70 \mathrm{~cm}$ e 2,0L de volume de trabalho. O sistema de dispersão de gases do reator constituiu em um difusor de ar de $1,5 \mathrm{~cm}$ localizado no centro da base da coluna. As condições de cultivo foram: concentração celular inicial de $100 \mathrm{mg} / \mathrm{L}$; temperatura de $26^{\circ} \mathrm{C}$; aeração de $1 \mathrm{VVM}$ (volume de ar por volume de meio por minuto) com a injeção de ar enriquecido com $15 \%$ de dióxido de carbono e presença de luminosidade de $150 \mu \mathrm{molm}^{-2} \mathrm{~s}^{-1}$.

\subsection{Amostragem e Métodos Analítico}

A concentração celular foi avaliada gravimetricamente por filtração de um volume conhecido de meio de cultura através de um filtro de 0,45 $\mu \mathrm{m}$ (Millex FG, Billerica-MA, EUA) e secagem a $60^{\circ} \mathrm{C}$ até peso constante. A concentração lipídica total da biomassa foi determinado pelo método de Bligh e Dyer modificado (1959), utilizando a relação entre metanol, clorofórmio e água destilada de 2: 1: 0,8 (v/v/v).

\section{RESULTADOS E DISCUSSÃO}

A tabela 1 apresenta os parâmetros cinéticos das diferentes microalgas. Observa-se que a Chlorella vulgaris apresentou maiores resultados de concentração celular máxima (3640 $\mathrm{mg} / \mathrm{L})$, velocidade específica máxima de crescimento $\left(0,036 \mathrm{~h}^{-1}\right)$ e produtividade em biomassa (18,4 mg/L.h) seguido da Phormidium autumnale. Contudo, vale ressaltar a importância dos 
lipídeos em termos de produtividade lipídica, sendo que este representa um dos principais parâmetros em termos de viabilidade econômica.

Tabela 1- Parâmetros cinéticos das diferentes espécies de microalgas

\begin{tabular}{lcccc}
\hline Espécie & $\mathrm{X}_{\text {máx }}(\mathrm{mg} / \mathrm{L})$ & $\mu_{\operatorname{máx}}\left(\mathrm{h}^{-1}\right)$ & $\mathrm{TG}(\mathrm{h})$ & $\mathrm{Px}(\mathrm{mg} / \mathrm{L} . \mathrm{h})$ \\
\hline C. vulgaris & 3640 & 0,036 & 192 & 18,4 \\
A. microscopica Nägelli & 2340 & 0,032 & 192 & 11,6 \\
P. autumnale & 3160 & 0,035 & 216 & 14,2 \\
S. obliquus & 1425 & 0,029 & 192 & 6,9 \\
\hline
\end{tabular}

$\mathrm{X}_{\text {máx: }}$ concentração celular máxima $(\mathrm{mg} / \mathrm{L}) ; \mu_{\text {máx}}$ : velocidade especifica máxima de crescimento $\left(\mathrm{h}^{-1}\right)$; TG: tempo de geração $(\mathrm{h})$; PX: produtividade em biomassa (mg/L.h).

Os dados apresentados na tabela 2 exibem a potencialidade do uso de Phormidium autumnale para obtenção de lipídeos, uma vez que apresentou produtividade lipídica de 3,8 $\mathrm{mg} / \mathrm{L} . \mathrm{h}$ e teor lipídico de $27,0 \%$. A produtividade lipídica é o produto da produtividade em biomassa e do teor lipídico, e por essa razão, tem maior importância. Visto que, altas taxas de produtividade tornam estes lipídeos uma alternativa sustentável e rentável para produção de biocombustíveis (Harwati et al., 2012) e outros produtos de alto valor agregado.

A tabela 2- Teor lipídico e produtividade lipídica das diferentes espécies de microalgas

\begin{tabular}{ccc}
\hline Espécie & $\mathrm{L}(\%)$ & $\mathrm{P}_{\mathrm{L}}(\mathrm{mg} / \mathrm{L} . \mathrm{h})$ \\
\hline C. vulgaris & 17,7 & 3,25 \\
A. microscopica Nägelli & 20,5 & 2,3 \\
P. autumnale & 27,0 & 3,8 \\
S. obliquus & 23,1 & 1,59 \\
\hline
\end{tabular}

L: teor lipídico (\%); $\mathrm{P}_{\mathrm{L}}$ : produtividade lipídica (mg/L h).

\section{CONCLUSÃO}

Todas microalgas avaliadas apresentaram capacidade para produção de lipídeos. No entanto, o estudo empregando diferentes espécies demostrou melhor resultado no cultivo da Phormidium autumnale. O cultivo apresentou melhores desempenho de produtividade lipídica (3,8 mg/L.h) e teor lipídico (27,0\%).

\section{REFERÊNCIAS}

BLIGH, E. G.; DYER, J. W. A rapid method of total lipid extraction and purification. Canadian Journal of Biochemistry and Physiology., v.37, n.8, p.911-917, 1959.

HARWATI, T. U.; WILLKE, T.; VORLOP, K. D. Characterization of the lipid accumulation in a tropical freshwater microalgae Chlorococcum sp. Bioresource technology., v. 121, p. 54-60, 2012.

MARIANO, A. B.; RAMOS, L. P.; VARGAS, J. V. C.; PULlIAN, R., BALMANT, W., MORAIS, K. C. C.; BOSA, J. A.; DZUMAN, M. J.; ARANTES, A. C. C. Comparação de meios de cultivo autotróficos, mixotróficos e heterotróficos para produção de 
biomassa de microalga com foco em biocombustíveis e coprodutos. In: $5^{\circ}$ Congresso Internacional de Bioenergia. 2009.

MARONEZE, M. M., SIQUEIRA, S. F., VENDRUSCOLO, R. G., WAGNER, R., DE MENEZES, C. R., ZEPKA, L. Q., JACOB-LOPES, E. The role of photoperiods on the growth and lipid content of microalgae Scenedesmus obliquus (CPCC05) - A potential strategy to costs reduce. Bioresource technology., v. 219, p. 493-499, 2016.

RIPPKA, R.; DERUELlES, J., WATERBURY, J. B., HERDMAN, M., STANIER, R. Y. Generic assignments strain histories and properties of pure cultures of cyanobacteria. Journal of General Microbiology., v.111, n.1, p.61, 1979. 